\title{
Lieux de la loi, lieux du savoir : maîtriser le temps et l'espace des autopsies sous les Qing
}

Spatializing Law, Spatializing Knowledge : Governing Time and Space in Qing

China's Forensic Practice

法律之所在、知識之所在: 清代驗屍活動中的時間與空間管理

Xin-zhe Xie

\section{OpenEdition}

\section{Journals}

Édition électronique

URL : http://journals.openedition.org/extremeorient/610

DOI : 10.4000/extremeorient.610

ISBN : 978-2-84292-558-1

ISSN : 2108-7105

Éditeur

Presses universitaires de Vincennes

Édition imprimée

Date de publication : 21 novembre 2016

Pagination : 127-150

ISBN : 978-2-84292-556-7

ISSN : 0754-5010

\section{Référence électronique}

Xin-zhe Xie, «Lieux de la loi, lieux du savoir : maîtriser le temps et l'espace des autopsies sous les Qing ", Extrême-Orient Extrême-Occident [En ligne], 40 | 2016, mis en ligne le 21 novembre 2018, consulté le 22 avril 2019. URL : http://journals.openedition.org/extremeorient/610 ; DOI : 10.4000/ extremeorient.610 


\title{
Lieux de la loi, lieux du savoir : maîtriser le temps et l'espace des autopsies sous les Qing
}

\author{
Xie Xin-zhe
}

Les rapports d'enquête qualifiés de modèles de l'époque des Qing ont tendance à décrire une autopsie telle qu'elle est réalisée en stricte conformité avec la loi et sans aucun obstacle : le magistrat se rend sur les lieux avec la plus grande célérité ; les marques de blessures sont assez claires pour être interprétées sans provoquer de controverse ; la description des blessures s'accorde parfaitement aux caractéristiques de l'arme présumée ; le résultat de l'examen post mortem coïncide avec les témoignages et les dépositions ; l'auteur du crime passe à l'aveu sans protester ; toutes les personnes impliquées dans le crime signent de bon gré les déclarations garantissant leur consentement aux conclusions de l'autopsie. Or, de récentes études montrent que les autopsies ne se déroulaient pas de façon aussi lisse que le laissent entendre de tels « dossiers inattaquables ${ }^{1}{ }^{»}$. De par sa nature, la loi présente nécessairement un certain écart par rapport à son application effective. Dans le cas de figure qu'est la pratique de l'autopsie, l'article 412 du code des Qing, intitulé « Ne pas réaliser une autopsie en conformité avec les faits » (jianyan shishang buyishi), le seul article régissant les autopsies, ne se soucie guère des situations particulières susceptibles d'empêcher l'observation rigoureuse des règles. Ces situations sont non seulement caractérisées par les lieux et les circonstances du crime, mais aussi par des facteurs encore plus imprévisibles et générateurs d'incertitude, comme la disponibilité des enquêteurs au moment des faits, leurs idiosyncrasies ainsi que la personnalité des personnages impliqués dans l'enquête.

1. Will 2015 : 14. Il existe des compilations des rapports d'enquête considérés comme exemplaires pour les magistrats peu expérimentés, par exemple le Xing'an chengshi et le Xue'an chumo. 
À part de tels obstacles qu'on peut qualifier d'externes, qui ont été suffisamment révélés par les travaux existants ${ }^{2}$, il existe en réalité une dimension intrinsèque au problème du cadre réglementaire des autopsies qui tenait à sa complexité et à son incohérence interne. L'article 412 du code regroupe un ensemble de règlements ayant directement trait à l'autopsie. En revanche, dans les faits, le cadre réglementaire des autopsies ne saurait se confiner à ce groupe de lois : des dispositions a priori sans rapport avec les autopsies étaient souvent invoquées pour déterminer les sanctions de fautes commises dans leur exécution. Sans parler de certaines démarches détaillées, qui donnent souvent l'impression d'être en flottement entre des obligations et des pratiques habituelles, qui ne sont pas abordées par l'article 412 et ses articles additionnels.

La provenance disparate des normes relatives à l'autopsie posait problème quant à leur interprétation. Il existait en effet des marges d'incertitude, créées par la possibilité d'apprécier les règles de façon variée. Par ailleurs, des amendements ajoutés en permanence au cadre réglementaire des autopsies augmentaient encore sa complexité, et son évolution législative (ses phases de débat, de tergiversation et de résistance) montre justement qu'il y avait un conflit entre certains principes de loi. Selon le principe auquel on se tenait, la position différait.

Pour donner un exemple concret des enjeux qui viennent d'être esquissés, nous nous focaliserons sur les règles visant à relever le défi posé à la réalisation des autopsies par le temps et l'espace. En effet, les historiens ont mis en lumière l'exigence de l'efficacité dans l'administration qui était hautement institutionnalisée sous les Qing ${ }^{3}$. Ce défi du temps, l'examen post mortem y était particulièrement confronté en raison de la vulnérabilité du support des preuves matérielles recherchées par l'autopsie, à savoir un cadavre voué à la dégradation. À cela s'ajoutait la contrainte spatiale, qui ne pouvait que ralentir l'accomplissement de la tâche. Les délais se trouvaient allongés par la durée mise par l'enquêteur habilité pour arriver sur les lieux du crime. Les dispositifs visant à gérer le temps et l'espace tiennent donc une place importante dans le cadre réglementaire des autopsies sous les Qing. Au terme d'une investigation sur l'évolution de ces dispositifs, nous analyserons la notion d'expertise très particulière telle qu'elle ressort des autopsies sous les Qing.

2. Will 2015.

3. Will 1989 : 98. 


\section{Les délais légaux}

\section{L'affaire du cadavre perdu}

Dans la nuit du $29^{\mathrm{e}}$ jour du $7^{\mathrm{e}}$ mois de l'année 4 du règne de Qianlong (1739), des dizaines d'hommes armés firent irruption dans le territoire de la sous-préfecture de Nanchuan, au Sichuan. Leur meneur, un dénommé Lei Qilong, prétendait avoir des droits sur le terrain qu'un groupe d'habitants de Nanchuan venaient d'acheter collectivement à un certain Yuan. L'altercation fit un grand nombre de blessés du côté des acquéreurs du terrain. L'un d'entre eux, Gong Wenbang, décéda de ses blessures ${ }^{4}$.

Le magistrat de Nanchuan était en mission à ce moment-là. C'est donc son homologue de la sous-préfecture voisine de Changshou qui vint sur place pour superviser l'autopsie. Or, au moins six jours s'écoulèrent entre les faits et l'autopsie, un délai suffisant pour que le corps de Gong commence à présenter des signes de dégradation sévère. Le magistrat de Changshou, jugeant un examen visuel de la surface du corps impossible, demanda l'autorisation de procéder à un examen des ossements.

De retour dix-sept jours après l'intervention de son collègue, le magistrat de Nanchuan traîna des pieds pour examiner les ossements de Gong. Par la suite, deux individus impliqués dans l'agression, dont Lei, furent successivement arrêtés et ne tardèrent pas à passer aux aveux. Pourtant, le magistrat continua à ne pas se soucier de réaliser l'examen du squelette, alors que l'affaire était pratiquement tirée au clair grâce aux aveux des coupables. L'examen des ossements, qui attendait depuis presque deux mois, aurait permis de mettre en place les dernières pièces du puzzle et de classer l'affaire.

Quoi qu'il en soit, le magistrat continua de faire preuve d'une certaine inertie, jusqu'au jour où, trois mois après son retour, le chef de village (dibao) signala la disparition du cadavre. Saisi par la gravité de la situation et craignant que la perte du cadavre ne vienne à se savoir, le magistrat prit les devants en prétendant qu'il attendait toujours de pouvoir emprunter un agent légiste expérimenté, raison pour laquelle il n'avait pu pratiquer l'examen des ossements.

Plus étonnant encore, il s'ingénia à dissimuler sa faute en désignant un autre agresseur, qu'il disait toujours en fuite, comme coupable principal de la mort de Gong, sans tenir compte du fait que Lei avait déjà avoué être l'auteur du coup fatal. En ouvrant cette nouvelle piste, le magistrat était en droit de demander qu'on fasse repartir le délai pour la clôture de l'affaire au moment

4. Neige daku dang'an (ci-après Neige daku) : $\mathrm{n}^{\circ} 065101$. 
de l'arrestation du fugitif. De ce fait, « le dossier était suspendu et ne pouvait pas être classé ». Tant que le magistrat tardait à mettre la main sur le coupable principal présumé, il avait un prétexte pour justifier de ne pas procéder à l'examen des ossements, et pouvait espérer que la mystérieuse disparition du cadavre demeure ignorée de ses supérieurs. Il s'en tira ainsi durant plus d'un an, jusqu'au jour où le gouvernement central décida de le renvoyer pour avoir « pris la vie du peuple avec désinvolture».

Les manigances du magistrat mettent en lumière sa bonne connaissance des réglementations relatives aux délais légaux. Si ses manœuvres parvinrent à le préserver de toute accusation pendant plus d'un an, c'est bien que les prétextes qu'il avançait étaient légalement fondés ou tout au moins légalement acceptables. D'abord, l'attente d'un agent légiste expérimenté semble avoir été une pratique courante qui ne fut pas pointée du doigt au cours de la procédure. Ensuite, la dérogation permettant de faire courir le délai légal pour clore un homicide à compter du moment de la comparution du prévenu principal faisait partie des règlements formellement établis. Autrement dit, tout en s'efforçant de dissimuler des fautes originellement dues à sa négligence et à sa désinvolture, le magistrat montrait sa parfaite connaissance du détail des règles ainsi que son habileté à en sélectionner les plus adaptées de manière à se protéger.

\section{La mésaventure de Wang Huizu}

De par sa nature même, la source qui relate l'affaire précédente ne laisse inévitablement entendre que la voix du gouverneur, indigné par l'abominable faute de son subordonné. Le cas cité ci-après a le mérite de nous permettre d'assister au «marchandage » entre un fonctionnaire local sur le banc des accusés et ses supérieurs ${ }^{5}$. Le débat, où alternent réprimandes et justifications, est là encore axé sur les modalités de calcul, au jour près, du délai légal. Le héros de l'affaire n'est autre que Wang Huizu (1730-1807), éminent auteur de manuels de fonctionnaires et incarnation du magistrat modèle. Il est intéressant de remarquer que Wang met en avant un argument identique à l'un des subterfuges employés par le magistrat de Nanchuan. Qu'un fonctionnaire réputé brillant et consciencieux s'en soit remis à la même justification qu'un magistrat qualifié de «désinvolte » a de quoi surprendre. Tout compte fait, qu'est-ce qui différencie ces deux fonctionnaires déchus, si ce n'est que nous disposons pour le second de son propre récit, alors qu'il est impossible

5. Will $2015: 32-35$. Will relate ce cas pour illustrer les représentations qu'avaient les fonctionnaires locaux de leur travail d'autopsie. 
d'entendre la voix du premier dans l'unique source qui relate les irrégularités qui lui furent reprochées?

L'affaire se déroule en 1791, lorsque Wang, alors magistrat intérimaire de la sous-préfecture supérieure de Daozhou (Hunan), fut désigné pour examiner quatre squelettes dans la sous-préfecture de Guiyang. Comme un examen de squelette s'avérait très sophistiqué, inquiet de l'absence d'agent légiste suffisamment qualifié pour pareille tâche dans toute la préfecture, Wang adressa une demande d'emprunt d'agent légiste à d'autres circonscriptions ${ }^{6}$. Tant qu'il ne disposait pas de l'agent légiste idoine, Wang ne pouvait exécuter sa mission. Deux mois s'étaient écoulés quand Wang fut appelé dans une souspréfecture voisine pour une mission d'autopsie. Sur le chemin du retour, il se cassa la jambe. La souffrance était telle qu'il finit par demander à être relevé de ses fonctions. Peu convaincu, le juge provincial était persuadé que Wang avait demandé son congé pour se soustraire à sa mission à Guiyang, qu'il était censé avoir accomplie depuis quatre mois.

Mais Wang et ses supérieurs étaient en désaccord sur les modalités de calcul du délai légal. Le gouverneur provincial lui reprochait son incapacité à trouver un agent légiste en deux mois. De son côté, Wang s'abritait derrière la loi, tout en apportant des précisions sur la chronologie : «Du $6^{\mathrm{e}}$ jour du $10^{\mathrm{e}}$ mois, jour où j'ai reçu la mission de Guiyang, au $5^{\mathrm{e}}$ jour $\mathrm{du} 12^{\mathrm{e}}$ mois, lorsque j'ai effectué ce déplacement pour exécuter une autopsie à Jianghua, le délai prévu pour traiter un dossier criminel n'avait pas encore expiré. En outre, l'agent légiste ne s'était pas encore présenté, et il est alors d'usage d'accorder un prolongement du délai ${ }^{7}$. » Son explication fut réfutée car le gouverneur s'en tenait à la date où Wang avait fait une chute, à savoir le $7^{\mathrm{e}}$ jour $\mathrm{du} 12^{\mathrm{e}}$ mois, ce qui ajoutait deux jours au décompte de Wang. Autrement dit, le délai de deux mois était déjà dépassé à ce moment-là. Le gouverneur s'entêtait à douter de la bonne foi de Wang au sujet de sa demande de congé. Wang se défendit en invoquant le fait que «dans le cas d'un examen des ossements, il est de coutume de calculer le délai à partir du jour où l'agent légiste arrive ». À cet argument, le gouverneur ne prêta qu'une oreille réticente ${ }^{8}$. Wang fut radié de la fonction publique qu'il ne put jamais réintégrer jusqu'à sa mort en 1807.

6. Wang [1850] $1996: 661$.

7. Ibid.: 664 .

8. Les supérieurs de Wang, quoique peu enclins à lui prêter foi, n'ont à aucun moment fait de commentaire sur la question de l'emprunt d'agent légiste, alors que la pratique avait été prohibée en 1769 dans le Hunan. Voir Hunan shengli cheng'an : 20.21a-22a. Cela pourrait s'expliquer par le statut particulier des directives provinciales, qui n'étaient pas applicables de façon aussi systématique que les directives centrales, et dont la 


\section{Les délais légaux concernant l'homicide}

Les cas cités ci-dessus soulèvent la question du délai légal. En général, pour tout homicide ordinaire survenu en province, l'enquête et le procès devaient être bouclés en six mois : le magistrat local disposait de trois mois; le préfet, le juge provincial et le gouverneur provincial, disposaient chacun d'un mois pour réviser l'affaire ${ }^{9}$.

Des dispositions spéciales encadrent certains traitements exceptionnels. Le délai pouvait être réduit pour des crimes de nature particulière ${ }^{10}$. La loi précise également les conditions accordant une extension du délai. Selon l'état d'avancement d'un dossier au moment de la succession de poste, le délai dont jouissait le successeur variait ${ }^{11}$. Il était également possible de suspendre le délai lorsque le fonctionnaire était en déplacement professionnel ${ }^{12} \mathrm{ou}$ lorsqu'un justiciable était dans l'incapacité de se présenter ${ }^{13}$. Cela pourrait faire croire que l'administration judiciaire des Qing s'était dotée d'un système suffisamment sophistiqué pour assurer son bon fonctionnement. Pourtant, les lacunes semblent avoir abondé, surtout quand l'autopsie était en jeu. Celles-ci compliquaient d'autant plus la tâche des fonctionnaires qu'ils ne savaient pas à quoi s'en tenir en la matière. D'un côté, l'article $412^{14}$ insiste sur le strict respect du principe d'immédiateté (ji) pour la réalisation d'une autopsie, mais aucune consigne ne fixe le nombre de jours pour effectuer l'autopsie, contrairement aux délais imposés au traitement complet des dossiers judiciaires ${ }^{15}$.

violation n'aurait pas été opposable dans un mémoire de censure. Il est aussi à noter qu'à l'échelle nationale, les demandes d'emprunt d'agent légiste en vue d'un examen de squelette n'étaient pas rares. Une source de la fin des Qing enjoignait même aux tribunaux provinciaux d'emprunter des agents légistes aux tribunaux voisins plutôt que de solliciter le ministère des Peines. Qingdai gongzhongdang zouzhe ji junjichu zhejian (ci-après GZD) : $\mathrm{n}^{\circ} 125668$.

9. Da Qing huidian shili [1899]1996 : vol. 800, 142.

10. Selon un arrêté de 1763 , en cas de meurtre commis par des inférieurs sur leurs supérieurs, ou dans le cas où le nombre des victimes atteignait trois ou quatre, le délai imparti était de deux mois (ibid. : 147). Dès 1800, pour tout meurtre grave qui entraînait l'exécution immédiate du condamné, le délai s'étendait à quatre mois au maximum (ibid. : 150-151).

11. Ibid. : 144-5.

12. Ibid.: 145.

13. Ibid.

14. La numérotation des lois et des articles additionnels suit celle établie par Huang Jingjia dans son édition du Duli cunyi de Xue Yunsheng (voir Xue 1905).

15. Un décret promulgué en 1722, puis modifié en 1806, autorise à calculer le délai limite pour résoudre une affaire criminelle à partir de la date de l'exhumation judiciaire 
Ce vide juridique était sans doute volontaire, car un délai maximal formellement fixé aurait pu compromettre le principe d'immédiateté, et par conséquent aurait laissé la possibilité au fonctionnaire de ne pas procéder à l'autopsie sitôt la plainte déposée. En 1755, remarquant que cet état de fait permettait aux magistrats de laisser traîner les exhumations judiciaires, Qingfu (1710- ?), le juge provincial du Hu-Guang, proposa de fixer un délai limite précis ${ }^{16}$. Le ministère des Peines objecta qu'en recevant l'ordre d'exhumation, le magistrat « est tenu de procéder à l'autopsie avec la plus grande célérité [...], il n'y a aucune raison de laisser [le magistrat] traîner les choses sous de quelconques prétextes, et c'est pour cela que les règles établies n'ont pas fixé de délai ». La proposition de Qingfu revenait à « vouloir fixer rigoureusement des délais, tout en favorisant au contraire les retards ».

L'inconvénient du flou sur la question du délai pour l'autopsie résidait dans la possibilité d'apprécier la situation de manière différente. Les supérieurs de Wang Huizu s'en tenaient manifestement au principe d'immédiateté énoncé à l'article 412. De son côté, Wang s'en remettait aux dispositifs sur les délais légaux, qui n'abordent pas de front l'autopsie. L'argumentation de Wang se rapproche donc de la stratégie du magistrat de Nanchuan dans le cas de 1739. En invoquant des motifs tels que l'attente d'un agent légiste et la capture du suspect principal, ce magistrat était parvenu à repousser le délai pour la résolution de l'affaire, de sorte que la procédure d'autopsie soit suspendue et que la disparition du cadavre ne s'ébruite pas. Selon son calcul, le respect du délai pour la clôture des affaires d'homicide l'emportait sur le principe d'immédiateté dans l'exécution d'une autopsie. La complexité des normes implique donc une dimension herméneutique, dans le sens où chacun pouvait tenir pour fondamentaux des principes différents.

\section{Gérer le temps et l'espace des autopsies}

Les délais légaux tout comme la diligence exigée par l'article 412 traduisent la pression du temps à laquelle étaient soumis les fonctionnaires sous les Qing en lien avec les autopsies. Si le signalement d'un décès anormal signifie le début d'une course contre la montre, un autre principe complique encore le défi,

(kaijian). Mais il ne précise pas où se situe le début du délai pour l'exhumation judiciaire.

16. Junjichu lufu zouzhe (ci-après LFZZ) : 3-1386, $\mathrm{n}^{\circ} 42$ et Neige daku : $\mathrm{n}^{\circ} 042358$. 
à savoir la compétence exclusive du magistrat ${ }^{17}$, qui est également primordiale dans la législation des Qing. L'interdiction faite à tout agent du yamen autre que le fonctionnaire désigné par la loi de superviser une autopsie existait déjà dans les institutions des époques précédentes. Mais la restriction s'est durcie sous les Qing : l'ordre d'attribution de l'habilitation à superviser une autopsie ne suivait pas la hiérarchie des grades des fonctionnaires sous les Song ${ }^{18}$ et aucun ordre de priorité ne semblait s'appliquer parmi les fonctionnaires statutaires sous les Yuan et les Ming ${ }^{19}$.

Ainsi, la loi des Qing témoigne d'une plus grande rigueur dans la hiérarchie des postes habilités à superviser les autopsies. Les législateurs des Qing durent payer le prix d'une telle restriction. Dans les faits, le principe de compétence exclusive du magistrat était grevé par un « cahier des charges » extrêmement lourd et la superficie très vaste de certaines circonscriptions. En conséquence, le casse-tête constitué par la pression du temps, de la distance et la compétence exclusive du magistrat eut pour résultat une inflation des articles additionnels annexés à l'article 412, lesquelles traduisent une tentative d'adapter les normes à la réalité.

Ces articles additionnels $\left(\mathrm{n}^{\text {os }} 5,10,11,17,19,21\right)$ statuent tous sur la répartition des responsabilités en matière d'autopsies et ont tous pour objectif de réduire les risques liés à l'écoulement du temps. Ils constituent une souscatégorie particulière parmi les 21 articles additionnels de l'article 412. Que faire lorsque ni le magistrat ni celui d'une circonscription limitrophe n'était à même de venir exécuter l'autopsie sans délai ? En remédiant à ce problème, l'article additionnel 412-5 est le premier à déroger au principe de la compétence exclusive du magistrat. Il permet de déléguer l'autopsie à l'adjoint (zuo'er) lorsque le magistrat local est absent et lorsque la localité la plus proche est distante d'au moins 50 ou $60 l i^{20}$.

17. Les magistrats sont désignés dans les lois sur l'autopsie par le terme générique zhengyinguan (fonctionnaire principal en charge du sceau). Gong 2006 : 172.

18. Liu $2005: 29-34,113-115$. Les autopsies relevaient par défaut du ressort du xianwei (lieutenant de sous-préfecture). Ce n'est qu'en dernier recours que l'on sollicitait le magistrat pour conduire une autopsie, Song huiyao 1996 : vol. 784, 536.

19. Wang Yu 1996: 508, 510. Un décret de 1339 dénonce une situation où les fonctionnaires statutaires se renvoyaient la balle pour échapper à une mission d'autopsie : Huang Ming tiaofa shilei zuan 1994 : vol. 5,903-4.

20. Sur la structure de l'administration locale sous les Qing, voir Ch'ü 1962 : 8-9. 


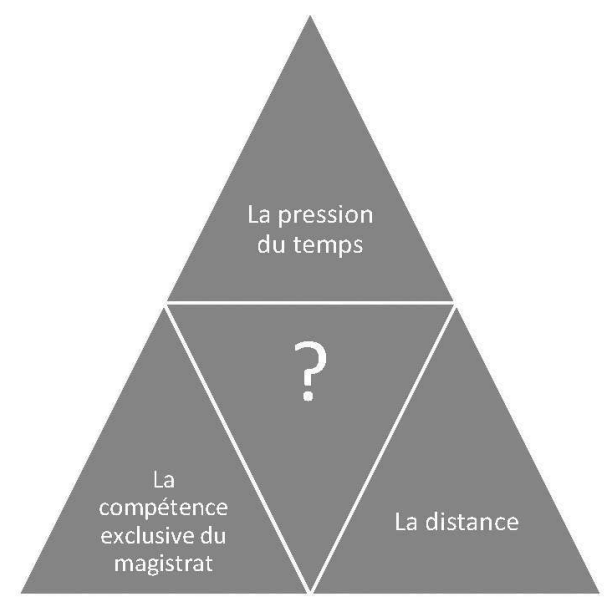

Schéma 1. Quelle norme pour concilier trois contraintes objectives?

L'article 412-10 prend acte de la difficulté engendrée par le fait que certaines sous-préfectures du Guizhou et du Sichuan ne disposaient pas de fonctionnaires adjoints, ou envoyaient ces derniers dans des postes éloignés du chef-lieu de la sous-préfecture. L'article 412-10 autorisait la délégation de l'autopsie à un agent ayant statut de "chef des services » (shoulingguan), qui avait une position intermédiaire entre, d'une part, les fonctionnaires et leurs adjoints et, d'autre part, les subalternes dans le gouvernement local ${ }^{21}$. L'administration territoriale était en effet assurée par deux types d'agents dont le statut était profondément différent : seuls les fonctionnaires en charge du sceau et leurs adjoints étaient membres de la fonction publique et dépositaires de l'autorité, tandis que leurs subordonnés recrutés localement devaient se borner à des tâches d'exécution. L'article 412-10 autorisait donc une entorse considérable à l'idéal de la compétence exclusive du magistrat, en concédant la responsabilité des autopsies aux fonctionnaires de grade inférieur d'un échelon. Néanmoins, précise le même article additionnel, la responsabilité du chef des services se limitait à établir une liste des lésions (shangdan), qui engageait moins de responsabilité que celles d'un adjoint.

21. Cette catégorie comprend le chef des registres (jingli), le chargé d'affaires (zhishi), le chef des commis du secrétariat (limu) et le chef de la prison (dianshi). Ch'ü 1962 : 8-9, 10 et passim. 
L'article additionnel 412-11 continue à étendre l'habilitation vers les échelons inférieurs. Elle autorise le chef de bureau de police local (xunjian), un poste subalterne chargé de la surveillance des lieux reculés, à exécuter l'autopsie en l'absence du magistrat et à défaut de fonctionnaire adjoint. L'intervention du chef de la police est aussi limitée à l'établissement de la liste des lésions et nécessite un examen de contrôle effectué a posteriori par un magistrat. L'article 412-17 dispose qu'au Guizhou, cet examen de contrôle peut ne pas avoir lieu si le magistrat ne peut se rendre sur place sous peu. L'article 412-19 marque un pas encore plus significatif dans la dérogation au principe de la compétence exclusive du magistrat. Tandis que jusqu'à présent, l'absence du magistrat constituait la condition sine qua non pour autoriser des exceptions, cette loi lève la restriction pour quelques zones isolées du Guangxi, où dorénavant, les adjoints en poste sur place procèdent à l'autopsie par défaut. L'article additionnel 412-21 élargit quant à elle le champ d'application de l'article additionnel 412-19 à trois sous-préfectures supérieures (ting) de la province du Fengtian.

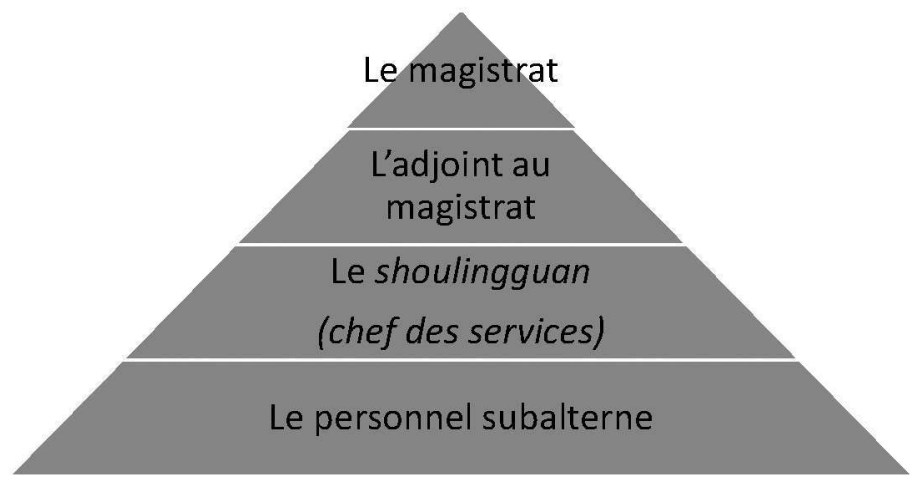

Schéma 2. Organigramme du gouvernement local (sous-préfecture et sous-préfecture supérieure)

Il se dégage donc une tendance vers plus de souplesse et davantage de liberté à l'égard de la compétence exclusive du magistrat. Le permis d'autopsier est accordé à des fonctionnaires de moins en moins importants et la condition de l'absence du magistrat finit par être levée. Ces concessions pour compenser la perte de temps engendrée par la distance montrent que le principe d'immédiateté finit par l'emporter sur la nécessité d'expertise. 


\section{Le processus législatif des réformes}

Il serait néanmoins illusoire de croire que le processus législatif qui a débouché sur cet ensemble de réglementations fut à sens unique. La nature et la forme des lois présentées ci-dessus tendent à faire oublier la phase de débat qui a précédé la codification de celles-ci. Puisqu'elles transcrivent uniquement des décisions prises en bout de chaîne, ces articles additionnels réduisent inévitablement au silence les « voix » des différentes opinions exprimées quand elles n'étaient encore que discutées. Il est donc nécessaire de se plonger dans les mémoires impériaux afin de connaître les arguments ayant nourri la délibération législative, si l'on veut comprendre comment de nouvelles idées émergeaient des circonstances pratiques et dans quelles circonstances les autorités pouvaient y opposer leur veto.

\section{- L'article additionnel 412-5}

L'article additionnel 412-5 est à cet égard un bon sujet d'étude. D'après Xue Yunsheng (1820-1901), cet article, annexé au code en 1740, tire son origine d'un mémoire de 1735 rédigé par le gouverneur du Guangxi, Jin Hong (1678-1740). Quoiqu'introuvable, le mémoire de Jin est cité dans un mémoire de $1737^{22}$ qui nous apprend que sa requête initiale fut motivée par la chaleur caniculaire et la vaste superficie des sous-préfectures de l'Ouest du Guangxi. En l'absence du magistrat, il proposait d'ordonner directement, à l'adjoint d'effectuer l'autopsie, sans qu'il fût besoin de solliciter le magistrat d'une localité limitrophe.

La requête de Jin prévoyait moins de restrictions que ce qui figura en définitive dans l'article additionnel 412-5. Les contraintes qui furent ajoutées au « prototype » déposé par Jin proviennent d'une proposition adressée en 1736 par Huang Shulin (1672-1756), juge provincial du Shandong ${ }^{23}$. Cette proposition, également citée dans le même mémoire de 1737, ne présente guère de différences avec l'article additionnel dans sa formulation finale. Cela établi, Huang suggérait d'imposer une condition de distance entre deux circonscriptions afin que l'adjoint puisse prendre directement en main l'autopsie en cas d'absence du magistrat.

Le mémoire de 1737, rédigé par Zhang Yunsui (1693-1751), gouverneur du Yunnan, mettait justement en question l'applicabilité du programme de Huang. Pour Zhang, le besoin de mesures accommodantes découlait autant

22. LFZZ : 3-1194, n 7 .

23. Wu Tan [1886] $1992: 1101$. 
des caractéristiques géographiques que des différences de gravité entre les circonstances entourant un décès. Au Yunnan, les sous-préfectures les plus étendues pouvaient avoir jusqu'à $1000 \mathrm{li}$ de largeur $(500 \mathrm{~km})$, tandis que même les plus petites s'étendaient sur 500 à 600 li. Qui plus est, en été, le climat caniculaire dans toute la province accélérait la putréfaction des cadavres. La réglementation en vigueur obligeant à signaler la mort au siège de la souspréfecture, même lorsqu'il fallait faire venir le magistrat d'une autre localité, les temps de communication et de déplacement retardaient énormément l'autopsie. Ainsi était-il à redouter de devoir en passer par l'examen des ossements, car la décomposition avancée des cadavres rendait impossible un simple examen des blessures sur la surface du corps.

Ensuite, Zhang évoque la propension chez «les sots Yi du Yunnan » (diansheng yuyi) à mettre un terme à leur vie au moindre incident, aussi trivial fut-il. Les suicides étaient donc très fréquents. En outre, les morts de maladie étaient nombreux chez les mineurs atteints par la fumée des fours et les marchands itinérants emportés par les miasmes. Dans ce contexte particulier, exécuter les autopsies conformément aux règles en vigueur semblait d'autant plus difficile que de lourdes charges pesaient par ailleurs sur les magistrats. Ainsi, quelques accommodements à la loi avaient été successivement approuvés par le ministère pour un petit nombre de circonscriptions, de sorte que certains adjoints, en poste loin du chef-lieu, puissent procéder directement à l'autopsie dans leur juridiction. Zhang entreprit d'étendre le champ d'application de ces dispositifs exceptionnels à d'autres circonscriptions ayant le même profil, en distinguant les cas d'homicide des décès non criminels. La procédure proposée par Huang Shulin s'appliquerait aux homicides tandis que pour les autres cas, il suffirait de procéder selon le projet de Jin Hong, c'est-à-dire de rendre systématiquement les adjoints responsables de l'autopsie en l'absence du magistrat.

L'amendement proposé par Zhang se situait donc à mi-chemin entre les propositions de Jin Hong et celles de Huang Shulin. Zhang n'envisageait de réserver les côtés les plus radicaux du programme de Jin qu'aux morts incontestablement non criminelles. De plus, à en croire Zhang, au début du règne de Qianlong, dans certaines régions du Yunnan, on se dispensait déjà de se conformer à la condition préalable de l'absence du magistrat, une pratique qui ne fut pourtant entérinée officiellement que quelques décennies plus tard avec l'article additionnel 412-19. 
- Caractéristiques locales en jeu:

les articles additionnels 412-19 et 412-10 et 11

En effet, les particularités territoriales d'ordre administratif aussi bien que géographique constituaient l'un des arguments privilégiés des fonctionnaires provinciaux pour proposer de reconsidérer les règles procédurales sur les autopsies. En plus des conditions météorologiques et géographiques, l'article additionnel 412-19 place les discussions relatives aux règles sur l'autopsie dans le contexte de l'évolution de l'administration locale.

Le mémoire à l'origine de l'article additionnel 412-19 date de 1763. Il est l'œuvre du juge provincial du Guangxi, Bokun (1724-1776) ${ }^{24}$. Ce mémoire portait sur Tian'e, une localité située au cœur des montagnes abruptes, dans la sous-préfecture de Lingyun (rattachée à la préfecture de Sicheng), et où l'adjoint du magistrat de Lingyun était installé. Tian'e étant distante du siège de la sous-préfecture d'au moins $300 l i$, et la température y étant habituellement élevée, Bokun craignait que faire dépendre l'intervention de l'adjoint de l'absence du magistrat ne provoque des retards et ne compromette les résultats des autopsies. D'où sa proposition que l'adjoint soit automatiquement habilité à effectuer les autopsies à Tian'e, que le magistrat soit présent ou non en son chef-lieu de Lingyun.

Pour étayer sa proposition, Bokun passait en revue l'histoire administrative du lieu. Créée en 1727 dans le cadre du programme d' " intégration des chefferies indigènes au système d'administration régulier » (tufu gailiu), la préfecture de Sicheng n'avait pas été divisée en sous-préfectures. Le préfet était obligé de traiter l'ensemble des dossiers judiciaires sur un territoire vaste. Il avait fallu attendre 1733 pour qu'on nomme un chef des registres (jingli) à Tian'e afin de relayer le préfet de Sicheng pour les litiges civils, les autopsies et les affaires de banditisme. Ce n'est qu'après la création de la sous-préfecture de Lingyun, en 1738, que le chef des registres fut retiré de Tian'e pour laisser la place à l'adjoint de la sous-préfecture nouvellement établie. Dès lors, les autopsies furent dévolues exclusivement au magistrat de Lingyun, son adjoint à Tian'e s'occupant uniquement des litiges ordinaires. Par cet exposé récapitulatif, Bokun voulait rappeler qu'un chef des registres avait déjà eu compétence en matière d'autopsie. «Un adjoint ayant un grade et un degré équivalents à ceux d'un chef des registres », il n'y avait donc aucune raison de refuser sa proposition. Qui plus est, le poste d'adjoint à Tian'e était pourvu selon un mode de sélection spécial qui garantissait que son détenteur

24. Neige daku $: \mathrm{n}^{\circ} 196715$. 
disposait d'une certaine expérience dans l'administration ${ }^{25}$. Par ces arguments, Bokun cherchait à démontrer que la suspension de la condition de l'absence du magistrat n'avait rien de choquant dans ce cas précis.

À vrai dire, le processus de réforme était fréquemment initié par des mesures locales, en vigueur dans un espace délimité. Les nouvelles dispositions voyaient ensuite leur champ d'application s'étendre, pour aboutir parfois à une généralisation à l'échelle nationale. C'est le cas des articles additionnels 41210 et 412-11. Le premier, d'après Xue Yunsheng, fut promulgué en 1751 et provient d'un mémoire de 1747 émanant du juge provincial du Guizhou, Jie Xizhou (?-1765) ${ }^{26}$. En plus de justifications centrées sur la grande superficie de certaines sous-préfectures et les chemins difficiles qui parsemaient toute la province, Jie prenait spécifiquement en compte le cas des localités dépourvues de fonctionnaire adjoint ou dont les adjoints étaient en poste loin du siège de la sous-préfecture. Dans ces deux cas, la souplesse assurée par l'article additionnel 412-5 n'était plus suffisante, d'où l'idée de confier la tâche aux chefs des services.

Cette proposition n'avait en fait rien d'inédit puisque Jie se référait à des pratiques déjà en vigueur dans la province du Gansu. Deux mémoires, datés de $1740^{27}$ et de $1746^{28}$, en attestent. Ils font mention de la permission spéciale accordée en 1738 au chef de service de procéder à l'autopsie dans les souspréfectures dépourvues d'adjoint en cas d'absence du magistrat. Dans ces deux mémoires, comme dans celui de Jie, cette prérogative de 1738 sert à étayer le bien-fondé des propositions qu'ils soumirent.

Le mémoire de 1746 fut adressé par Suchang (?-1768), le préfet de Fengtian, qui faisait valoir que dans sa juridiction, rares étaient les sous-préfectures dotées d'adjoints. En outre, la distance souvent importante entre les chefs-lieux ne permettait pas de trouver aisément un homologue disponible à proximité lorsque le magistrat était absent, sans parler des éventuels empêchements que pouvaient avoir les magistrats des alentours. Or, la plupart des morts étaient des suicides ou des décès naturels, les défunts étant souvent des occupants d'auberges ou des gens embauchés pour des travaux de force. En conséquence,

25. Bokun évoque la « sélection pour la mutation » (jianxuan diaobu). Cette modalité particulière de recrutement s'appliquait à des circonscriptions jugées difficiles à gérer où, au lieu d'attribuer les postes à des fonctionnaires inexpérimentés proposés par le ministère du Personnel, il appartenait au gouverneur provincial de sélectionner un fonctionnaire directement au sein des effectifs existants. Voir Guo 2003 : 295-296.

26. GZD : n 001259, Da Qing huidian shili [1899] 1996 : vol. 810, 373.

27. Neige daku: $\mathrm{n}^{\circ} 072695$.

28. LFZZ : $3-1382, n^{\circ} 20$. 
« les aubergistes ou les employeurs ne se risquaient à ensevelir les morts qu'après que l'autopsie eût tout élucidé », ce qui multipliait considérablement les interventions du magistrat. Afin de lui éviter des déplacements inutiles, et de sorte que sa présence fût mieux garantie dans les cas d'homicides, Suchang demandait d'appliquer dans sa préfecture le précédent du Gansu : en cas de suicide ou de décès imputable à la maladie, si le magistrat n'était pas disponible pour l'autopsie, il serait permis à un chef des services de le faire.

Le mémoire de 1740 invoquait lui aussi la dérogation de 1738, mais sans se restreindre à son cadre. Au lieu du chef des services, c'est au chef de la police que devaient être confiées les autopsies, et ce sans aucune condition. Son auteur, Fang Xian (?-1741), était le gouverneur intérimaire du Sichuan. Il faisait valoir que deux lieux-dits, Lerong et Pingding, se situaient particulièrement loin de la sous-préfecture supérieure de Xuyong à laquelle ils étaient pourtant rattachés. Aussi suggérait-t-il qu'il soit du ressort du chef de la police posté sur place de procéder à l'autopsie. La demande reçut un avis favorable et par la même occasion, un poste d'agent légiste fut créé sous l'autorité des chefs de la police. De ce fait, le dispositif de Fang Xian est, à notre connaissance, la plus ancienne expression formelle de l'esprit de l'article additionnel 412-11.

D'après Xue Yunsheng, l'article 412-11 tire son origine d'un mémoire adressé en 1753 par Suchang, mais cette fois-ci en tant que gouverneur du Guangdong. Son projet consistait à rendre plus contraignantes les modalités de la délégation aux chefs des services et aux chefs de la police en matière d'autopsies. Contrairement à la mesure exceptionnelle obtenue par Fang Xian pour le Sichuan, Suchang entendait imposer l'absence du magistrat comme condition nécessaire pour que le chef de la police puisse autopsier. Outre cela, comme Xue Yunsheng le remarque, à la différence des limites imposées par l'article 412-10 pour la responsabilité des chefs des services, l'article 412-11 exigeait que le magistrat effectue dès son retour un examen de contrôle (fuyan) afin de valider les premiers résultats ${ }^{29}$.

En dépit de ces garde-fous supplémentaires, la série de réformes initiée par Jin Hong suscitait une certaine inquiétude. On estimait que l'assouplissement du principe de la compétence exclusive du magistrat risquait de provoquer de nouveaux abus. C'est dans cet esprit qu'environ sept mois après la proposition de Suchang, le juge provincial du Jiangxi, Fan Tingkai (jinshi 1736), rédigea un mémoire exprimant ses réserves sur la portée territoriale de la mise en place de

29. Xue [1905] 1970 : 1271 ; Xue Yunsheng est probablement le seul à clarifier explicitement l'opposition entre la vérification documentaire (chayan tiange) dont parle l'article 412-10 et l'autopsie de contrôle (fuyan shishang) de l'article 412-11. 
la nouvelle mesure ${ }^{30}$ : Fan craignait que les accommodements se généralisent. Faute d'une définition précise de ce qu'était un « lieu isolé », le tribunal local pouvait aisément prétendre que la distance avec une circonscription limitrophe était trop grande pour faire venir un autre magistrat en remplacement du magistrat absent. D'ailleurs, le problème de la distance n'existait pas dans sa province où, d'après lui, les sous-préfectures « étaient disposées comme les pièces sur l'échiquier », distantes de quelques dizaines de $l i$, une centaine au maximum. Dans tous les cas, contrairement aux provinces initiatrices des réformes depuis 1735 (Guangxi, Gansu, Guizhou, Sichuan et Guangdong), il était possible pour un magistrat de se rendre dans une sous-préfecture voisine en une journée. Fan s'inquiétait aussi de la qualité des nombreux fonctionnaires de rang inférieur au magistrat. Dans sa juridiction, les adjoints doués avaient déjà obtenu une promotion au poste de magistrat intérimaire ou s'étaient vu régulièrement confier les responsabilités d'un magistrat. Les autres ne faisaient preuve d'aucun talent particulier, sans parler des chefs des services et des subalternes, au sujet desquels «plus le grade est bas, plus la vertu est médiocre ».

Fan remettait donc en question non seulement la réforme en cours, mais aussi, de manière rétroactive, l'article additionnel 412-5, le pionnier des réformes. Cet article avait fixé une distance minimum de 50 ou de $60 \mathrm{li}$ entre deux circonscriptions pour autoriser les adjoints à pratiquer l'autopsie, une distance que Fan jugeait insuffisante. Appelé à donner son avis, le gouverneur général du Liangjiang, Errong'an (?-1755), souligna que les mesures accommodantes ne s'étaient jamais appliquées qu'à titre conditionnel ${ }^{31}$. Seules les provinces rencontrant des difficultés semblables à celles énoncées par la loi pouvaient se prévaloir des exceptions. Les mises en garde de Fan contre les excès de flexibilité étaient donc exagérées. Enfin, pour prévenir tout abus, Errong'an demanda tout de même qu'une distance minimale entre deux souspréfectures soit établie, solution approuvée par Qianlong ${ }^{32}$. Les scrupules conservateurs de Fan n'avaient donc pas déclenché de réactions d'ampleur parmi ses supérieurs et confrères. Néanmoins, cinq ans après, en 1758, une voix concordante s'éleva au Hunan, exprimant la même méfiance vis-à-vis de tous les agents auxiliaires pour les enquêtes sur la maladie des prisonniers et insistant sur le même principe de l'habilitation exclusive du magistrat ${ }^{33}$. Approuvée dans un premier temps, cette mesure fut remise en cause en 1763

30. LFZZ : 3-1384, $\mathrm{n}^{\circ} 37$.

31. GZD : $n^{\circ} 403004892$.

32. Gongzhongdang Qianlong chao zouzhe 1982 : 390 .

33. LFZZ : $3-1198, n^{\circ} 8$. 
par le juge provincial du Gansu, qui invoquait l'étendue particulière des circonscriptions de sa province ${ }^{34}$.

Ainsi, par contraste avec le Jiangxi et le Henan, c'est surtout dans les provinces situées aux confins de la Chine elle-même que s'exprimait le besoin de prévenir les risques de retard. C'est probablement dans ce contexte qu'en 1758 on reprocha au chef de la prison de la sous-préfecture de Qianyang, à l'extrémité sud-ouest du Hunan, d'avoir demandé au magistrat de Huitong de parcourir 360 li pour une autopsie, au lieu d'en charger le chef de la police qui était sur le lieu du crime. Surpris d'être sollicité, le magistrat de Huitong en conclut que les propositions de Suchang insérées dans le code des Qing seulement deux ans plus tôt n'étaient pas encore entrées en pratique. Le juge provincial ne comprenait pas non plus « pourquoi [la nouvelle règle] n'avait jamais été appliquée au Hunan, alors même que l'arrêté ministériel avait si clairement discuté et tranché ${ }^{35} »$. Au vu de l'insistance avec laquelle le juge provincial exigea que le dispositif flexible soit dûment appliqué, il ressort que la recherche d'efficacité prévalait sur le souci de fiabilité concernant les fonctionnaires auxiliaires.

\section{- L'examen de contrôle : l'article additionnel 412-17}

Si la tendance à l'assouplissement des normes a suscité des craintes chez certains comme Fan Tingkan, ceux-ci auraient probablement été encore plus choqués par la manière dont Pei Zongxi (1712-1779), le gouverneur du Guizhou lança dans un mémoire de 1776 l'innovation qui devait être codifiée sous forme de l'article additionnel 412-17. Pei déclara que la procédure de l'examen de contrôle, exigée par l'article 412-11, était absurde ${ }^{36}$. En effet, la loi n'autorisait les chefs des services et les subalternes à procéder à l'autopsie que lorsqu'aucun magistrat ne pouvait intervenir ; mais si aucun magistrat n'était en mesure de venir effectuer l'autopsie, il n'était pas plus à même de venir faire l'examen de contrôle. Dans les deux cas, le risque était grand que le magistrat ne puisse examiner la dépouille avant qu'elle ait atteint un degré de décomposition avancée, lorsque les marques générées par la putréfaction pouvaient être facilement confondues avec les véritables traces de blessure. L'autopsie de contrôle perdait ainsi sa raison d'être. Plutôt que confirmer ou invalider le résultat de la première autopsie, elle brouillait les causes du décès. En outre, puisque la moindre discordance entre les rapports d'autopsies

34. Ibid.

35. Hunan shengli cheng'an: 20.3b-6b.

36. LFZZ : 3-1421, n 28. 
rédigés pour une même affaire entrainait le rejet du dossier par les supérieurs, les magistrats se contentaient souvent, lors de leurs examens de contrôle, de déclarer «n'avoir relevé aucune différence [entre deux autopsies] ». Par conséquent, « l'examen de contrôle n'était plus qu'un mot vide de contenu ». Estimant que «maintenir en vigueur une formalité vide de sens ne présentait aucun avantage pour la justice », Pei demanda l'abolition universelle de l'examen de contrôle prévu par l'article additionnel 412-11.

Bien que l'empereur Qianlong trouvât que Pei « semble voir juste », au vu du contenu de l'article 412-17, sa proposition dut subir une restriction comme celle de Jin Hong : au lieu de l'abrogation pure et simple de l'examen de contrôle, il fut décidé d'opter pour une dispense conditionnelle. Dans la version finale de la loi, cette dispense ne fut autorisée qu'aux confins du Guizhou et durant la seule saison chaude.

\section{Conclusion}

\section{La question de l'expertise}

L'étude approfondie du processus législatif des règles sur les autopsies nous a permis de mieux percevoir la nature des compétences attendues des fonctionnaires pour l'autopsie en tant qu'institution plutôt que comme technique. Ces compétences allaient en fait au-delà du simple respect des règles. On exigeait également du fonctionnaire la capacité d'articuler ces règles avec d'autres types de normes, de savoir les interpréter, de maîtriser leur évolution dans le temps et de prendre conscience de leurs lacunes.

Ainsi, c'est plus une expertise bureaucratique qu'une connaissance savante que nous avons constatée chez les fonctionnaires en matière d'autopsie. Cette expertise bureaucratique est conforme à l'image idéalisée qu'on se faisait des lettrés-fonctionnaires, un mélange de facultés intellectuelles supérieures et de vertus hors du commun, qui expliquait l'indéfectible confiance placée en eux. Paradoxalement, même les compromis avec le principe de la compétence exclusive du magistrat reviennent à montrer l'importance de celui-ci. Sinon, les législateurs du dix-huitième siècle n'auraient pas posé autant de conditions pointilleuses à ces compromis, en engageant tout un raisonnement axé sur le temps et l'espace.

La prépondérance des considérations d'ordre éthique, enfin, transparaît dans les inquiétudes ressenties face aux réformes et à leur nombre croissant d'amendements. L'objection de Fan Tingkai qui désapprouvait la témérité des nouvelles mesures en est la meilleure illustration. Pour Fan, le statut de magistrat garantissait à lui seul le respect scrupuleux de la procédure, lequel 
concourait à juguler les abus et, en conséquence, à assurer la véracité des rapports d'autopsie. La formule sans ambages de Fan, «plus le grade est bas, plus médiocre est la vertu », exprime on ne peut plus clairement le lien entre fiabilité morale et position dans la hiérarchie mandarinale. On retrouve cette corrélation dans l'argument de Bokun, même si sa requête allait dans un sens complètement opposé. Son argument se fondait sur l'équivalence en grade entre le chef des registres et l'adjoint au magistrat. Ici encore, le raisonnement accordait crédibilité à un membre du gouvernement local à hauteur de sa position.

D'autres directives montrent à quel point ce mode de raisonnement était ancré dans la pensée bureaucratique autour des autopsies. Bien que la tendance à l'assouplissement réglementaire parût irréversible, des décrets à partir du milieu du dix-neuvième siècle exprimèrent le même souci que Fan. Par exemple, en 1884 , un décret ${ }^{37}$ interdit à certaines sous-préfectures de la province du Fengtian de déléguer l'autopsie à tout agent auxiliaire si l'autopsie devait être organisée à moins de 200 li du chef-lieu. Même s'il s'agissait d'une distance de plus de $200 \mathrm{li}$, le magistrat devait toujours pratiquer l'autopsie lorsque le tribunal était peu occupé. Des décrets promulgués en 1856 et en 1878 rappelèrent qu'il était strictement interdit de sous-traiter une autopsie à un scribe, un agent légiste, un militaire, voire un conseiller technique (тиуои) ${ }^{38}$.

Tout indique que ces prohibitions ne se souciaient point de la compétence technique puisqu'elles excluaient les agents légistes et les conseillers techniques, qui étaient pourtant souvent experts en technique d'autopsie. Partant, l'expertise particulière que détenaient les magistrats en matière d'autopsie, reposant sur leur qualité morale supposée supérieure, leur conférait ce que l'on pourrait appeler une autorité sans connaissance faisant autorité. Or, l'autorité exercée par les magistrats sur la scène des autopsies ne se bornait pas à un simple contrôle de la procédure ou du comportement de leurs subordonnés. Envisager le contraire ne rendrait pas justice à la complexité de la question de l'expertise dans le contexte de l'autopsie. Il existe une profusion de cas où le magistrat, sceptique, questionnait l'agent légiste sur les constatations post mortem que le dernier avait rapportées. L'agent légiste pouvait certes être de bonne foi. Mais le magistrat se faisait un devoir de passer au crible les faits relevés par l'agent légiste, de manière à délivrer une conclusion susceptible d'être acceptée sans controverse. Garante du rapport d'autopsie, l'intervention du magistrat avait également un impact sur les manières dont les états du monde avaient à être décrits, compris et exploités.

37. Da Qing huidian shili [1899] 1996 : vol. 810, p. 376.

38. Ibid. : 193 et Liu [1910] 1996 : vol. 819, 18. 
La moralité dont le magistrat était supposé être la personnification même non seulement lui procurait une autorité de droit mais aussi accréditait tout diagnostic post mortem revêtu de son approbation. Moralité et collecte des données étaient donc indissociables. Ce constat rappelle l'inséparabilité entre l'ordre social et l'ordre cognitif que Steven Shapin s'est employé à démontrer pour le contexte de l'Angleterre. Il a ainsi mis au jour le rôle décisif de la crédibilité et de la morale de la classe gentlemen dans les activités scientifiques du dix-septième siècle. La production du savoir, affirme-t-il, « a lieu dans un champ moral et implique des appréciations spécifiques sur la vertu et les caractéristiques des types de personnes ${ }^{39}$ ».

La question de l'expertise dans le contexte des autopsies chinoises présente assurément des similarités avec l'analyse de Shapin. Néanmoins, l'autopsie chinoise comporte une telle particularité qu'elle est irréductible à son modèle. Les observations post mortem participaient d'une décision qui engageait des vies humaines, tant celle de la victime (au nom de qui la justice devait être rétablie) que celle de l'inculpé (guetté par une mort punitive). Ainsi, à la croisée des faits juridiques (reconstitution du crime) et des faits d'observation (traces sur le cadavre), les autopsies dans la Chine impériale tardive constituaient un espace ouvert, traversé par des conflits d'intérêt et chargé de méfiance réciproque (tant entre la population locale et le magistrat qu'entre ce dernier et ses supérieurs). Cela fait écho au consentement obligatoire de toutes les parties au résultat de l'autopsie. En ce sens, l'autopsie chinoise ressemblait plus à un processus de négociation qu'à un diagnostic imposé par l'autorité d'un savoir spécialisé, apportant ainsi de nouveaux éléments à la réflexion sur la notion d'expertise.

\section{BIBLIOGRAPHIE}

CH'Ü, T'ung-tsu (1962). Local Government in China under the Ch'ing. Cambridge (Mass.) : Harvard University Press.

Da Qing huidian shili 大清會典事例 (Compendium des directives administratives des Grands Qing) (1899), compilé par Kungang 崑岡 et Liu Qiduan 劉啟端. In Xuxiu Siku quanshu 續修四庫全書. Shanghai : Shanghai guji chubanshe, 1996, vol. 798-814.

Gong, Yanming 龔延明 (2006) (éd.). Zhongguo lidai zhiguan bieming dacidian 中 國歷代職官別名大辭典 (Grand dictionnaire des noms alternatifs de postes de

39. « [...] takes place on a moral field and mobilizes particular appreciations of the virtues and characteristics of types of people.» $C f$. Shapin 1994 : xxvi. 
la fonction publique au cours de l'histoire chinoise). Shanghai : Shanghai cishu chubanshe.

Gongzhongdang Qianlong chao zouzhe 宮中檔乾隆朝奏摺 (Les mémoires impériaux du règne de Qianlong tirés des archives de la Cour). Taipei : Gugong bowuyuan, 1982-.

GuO, Chengkang 郭成康 (2003). Shiba shiji de Zhongguo zhengzhi 十八世紀的中國 政治 (La politique en Chine au XVIII ${ }^{\mathrm{e}}$ siècle). Taipei : Zhaoming chubanshe.

Huang Ming tiaofa shilei zuan 皇明條法事類纂 (Collection catégorisée des réglementations des augustes Ming), publié sous le règne de l'empereur Hongzhi 弘治(1487-1505). In Zhongguo zhenxi falü dianji jicheng 中國珍稀法律典籍集 成 (Compendium des textes judiciaires chinois rares). Pékin : Kexue chubanshe, 1994, série 2, vol. 4-6.

Hunan shengli cheng'an 湖南省例成案 (s.d.) (Les précédents provinciaux et les cas homologués du Hunan), conservé à Tōyō bunka kenkyūjo. Tokyo, cote : B3591800.

ILIBU 伊里布 (1881) (comp.). Xue’an chumo 學案初模 (Modèles élémentaires pour s'initier aux affaires judiciaires), conservé à Tōyō bunka kenkyūjo, Tokyo, cote : B3860400.

Junjichu lufu zouzhe 軍機處錄副奏摺 (Copies des mémoires au trône tenues par le Grand Conseil), conservé aux Archives historiques $n^{\circ} 1$ de Chine 中國第一歷 史檔案館.

LIU, Hsin-Chun 劉馨珺 (2005). Mingjing gaoxuan : Nan Song xianya de yusong 明鏡 高懸：南宋縣衙的獄訟 (La clairvoyance du juge : Les procès aux tribunaux des Song du Sud). Taipei : Wunan tushu chuban gongsi.

LIU, Jinzao 劉錦藻 (1910). Qing chao xu Wenxian tongkao 清朝續文獻通考 (Deuxième compilation des documents administratifs sous les Qing). In Xuxiu Siku quanshu, vol. 815-821.

LÜ, Zongli 呂宗力 (1999) (éd.). Zhongguo lidai guanzhi dacidian中國歷代官制大辭 典 (Grand dictionnaire du système de la fonction publique au cours de l'histoire chinoise). Pékin : Beijing chubanshe.

MENG, Hushi 孟瑴史 (1877) (comp.). Xing'an chengshi 刑案成式 (Modèles établis pour les affaires criminelles). Mochi shuwu 墨池書屋, conservé à la C.V. Starr East Asian Library, New York, cote : 4894.51354.

Neige daku dang'an 內閣大庫檔案 (Archives du Grand Secrétariat), conservé à l'Academia sinica, Taipei.

Qingdai gongzhongdang zouzhe ji junjichu zhejian 清代宮中檔奏摺及軍機處摺件 (Mémoires impériaux tirés des archives de la Cour des Qing, et documents du Grand Conseil), conservé au musée national du Palais de Taipei.

SHAPIN, Steven (1994). A Social History of Truth. Civility and Science in SeventeenthCentury England. Chicago : University of Chicago Press.

Song huiyao 宋會要 (Compilation des documents importants de la dynastie des Song). Révisé par Xu Song 徐淞 (1781-1848). In Xuxiu Siku quanshu, vol. 775-786. 
Xie Xin-zhe

WANG, Huizu 汪輝祖 (1796). Bingta menghen lu 病榻夢痕錄 (Traces de rêve au lit de malade) et la suite (Menghen yulu 夢痕餘錄), éd. de Gong Yu 龔裕, 1850. In Хихіи Siku quanshu, vol. 555 : 607-733.

WANG, Yu 王與 (1308). Wuyuan lu 無冤錄 (Le recueil pour anéantir l'injustice). In Хuxiu Siku quanshu, vol. 972 : 503-516.

WILL, Pierre-Étienne (1989). «Bureaucratie officielle et bureaucratie réelle ». Études chinoises, vol. 8.1 : 69-142.

WILL, Pierre-Étienne (2015). « Entre routine bureaucratique et passion du métier. Sur la pratique médicolégale en Chine à l'époque des Qing ». Cahier du centre de Pékin de l'École française d'Extrême-Orient, 17 : 1-41.

WU, Tan 吳壇 (1992 [1880]). Da Qing lüli tongkao jiaozhu 大清律例通考校注 (Étude exhaustive et annotée du Code des Grands Qing). Pékin : Zhongguo zhengfa daxue chubanshe.

XUE, Yunsheng 薛允昇 (1970 [1905]). Duli cunyi 讀例存疑 (Doutes persistants à la lecture des articles additionnels), édité par Huang Jingjia 黃静嘉. Taipei : Chinese Materials Center.

\title{
GLOSSAIRE
}

\author{
Bokun 柏琨 \\ diansheng yuyi 滇省愚彝 \\ dianshi 典史 \\ dibao 地保 \\ Errong'an 鄂容安 \\ Fan Tingkai 范廷楷 \\ Fang Xian 方顯 \\ fuyan shishang 覆驗屍傷 \\ fuyan 覆驗 \\ Huang Shulin 黃叔琳 \\ Huitong 會通 \\ $j i$ 即 \\ jianxuan diaobu 揀選調補 \\ jianyan shishang buyishi 檢驗屍傷不以實 \\ Jie Xizhou 介錫周 \\ Jin Hong 金鉷 \\ jingli 經歷 \\ kaijian 開檢 \\ Lerong 樂榮 \\ limu 吏目 \\ Lingyun 凌雲
}


muyou 幕友

Pei Zongxi 裴宗錫

Pingding 平定

Qianyang 黔陽

shangdan 傷單

shoulingguan 首領官

Sicheng 泗城

Suchang 蘇昌

Tian'e 天峨

ting 廳

tufu gailiu 土府改流

$x i a n w e i$ 縣尉

xunjian 巡檢

Xuyong 敘永

Zhang Yunsui 張允隨

zhengyinguan 正印官

$z h i s h i$ 知事

zuo'er 佐負 
